\title{
4th International LIS-EPI meeting: una mirada al futuro de la información
}

\author{
Por Natalia Arroyo-Vázquez y Javier Guallar
}

\begin{abstract}
Resumen: Informe sobre el 4th International LIS-EPI Meeting que tuvo lugar en Valencia en noviembre de 2009 bajo el lema "La información en 2015". Los temas que se trataron fueron el futuro del sector de la información y de las bibliotecas, las rich internet applications (RIAs), el software libre en bibliotecas, el acceso abierto y los dispositivos móviles.

Palabras clave: Congresos, Encuentros, LIS-EPI meeting, Bibliotecas, Información, Futuro profesional, Acceso abierto, Dispositivos móviles, Prospectiva.

Title: 4th International LIS-EPI meeting: a view on the future of information

Abstract: Report on the 4th International LIS-EPI Meeting which was held in Valencia in November 2009, under the title "Information in 2015". The topics covered were the future of information and libraries, Rich Internet Applications (RIAs), free software in libraries, open access, and mobile devices.

Keywords: Conferences, Meetings, LIS-EPI meeting, Libraries, Information, Professional future, Open access, Mobile devices, Prospective.
\end{abstract}

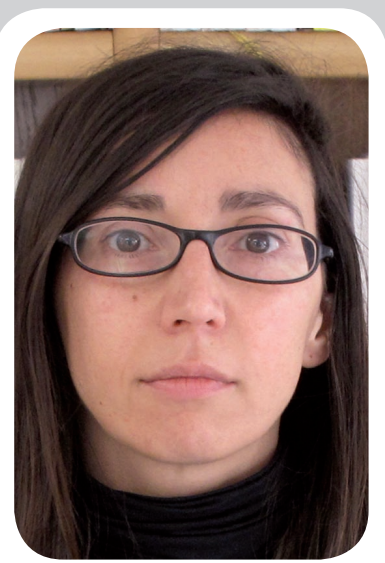

Natalia Arroyo-Vázquez es licenciada en documentación por la Universidad de Salamanca, grado y diploma de estudios avanzados. Trabaja como documentalista en el Departamento de Análisis y Estudios de la Fundación Germán Sánchez Ruipérez.

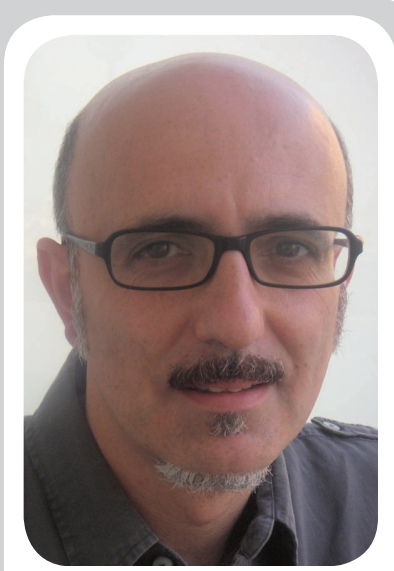

Javier Guallar es licenciado en geografía e historia por la UB, en información y documentación por la UOC y DEA por la UB. Es profesor de la UB, subdirector de El profesional de la información y coordinador del Anuario ThinkEPI.

Arroyo-Vázquez, Natalia; Guallar, Javier. "4th International LIS-EPI meeting: una mirada al futuro de la información”. El profesional de la información, 2010, enero-febrero, v. 19, n. 1, pp. 101-108.

DOI: 10.3145/epi.2010.ene.15

LOS DÍAS 26 Y 27 DE NOVIEMBRE de 2009 tuvo lugar en Valencia la cuarta edición del LIS-EPI meeting, organizado como la anterior (Redondo, 2008) por la Universidad Politécnica de Valencia (UPV), la Universitat Oberta de Catalunya (UOC), y la revista $E l$ profesional de la información (EPI).

Si las dos primeras ediciones del meeting en Ginebra 2005 y Valencia 2007 tuvieron como único tema central el acceso abierto, en 2008 se produjo un cambio de formato que se ha mantenido en 2009, con dos jornadas de conferencias, debates y talleres en torno a un eje común, que en la anterior ocasión fue la innovación y en ésta la prospectiva.

Bajo el título "La información en 2015" el encuentro proponía un debate con expertos de diferentes áreas del sector de la información sobre los derroteros por los que se entrevé que puede transitar nuestra profesión en los próximos años. Y durante esos días se puedo escuchar, ver y debatir parte de ese futuro que casi ya está aquí.

El evento tuvo presencia en las redes sociales con página web, blog, grupo en Facebook y perfil en Twitter, y fue además retransmitido en directo via Twitter por numerosos asistentes. Los contenidos se podrían agrupar de manera aproximada en cinco áreas: futuro de la información, bibliotecas y la profesión; rich internet applications (RIAs); software libre; acceso abierto; y dispositivos de acceso a la información.

\section{Futuro de la información y las bibliotecas}

El encuentro, que fue inaugurado por María-Antonia Carrato, subdirectora general de coordina- ción bibliotecaria, se inició con la visión desde fuera de nuestras fronteras de Eric Lease Morgan y Josef Herget en sendas conferencias. Morgan, director del Departamento de Acceso Digital y Arquitectura de la Información en las Bibliotecas de la Universidad de Notre Dame (EUA), destacó los cambios que se han producido en las bibliotecas en los últimos años con la llegada de la Web y de Google, que se ha convertido en el estándar de búsqueda de información para los usuarios.

“Los profesionales de la
información deberían tener
conocimientos de xml,
indexación, bases de datos
relacionales y algo de
programación" (Morgan)




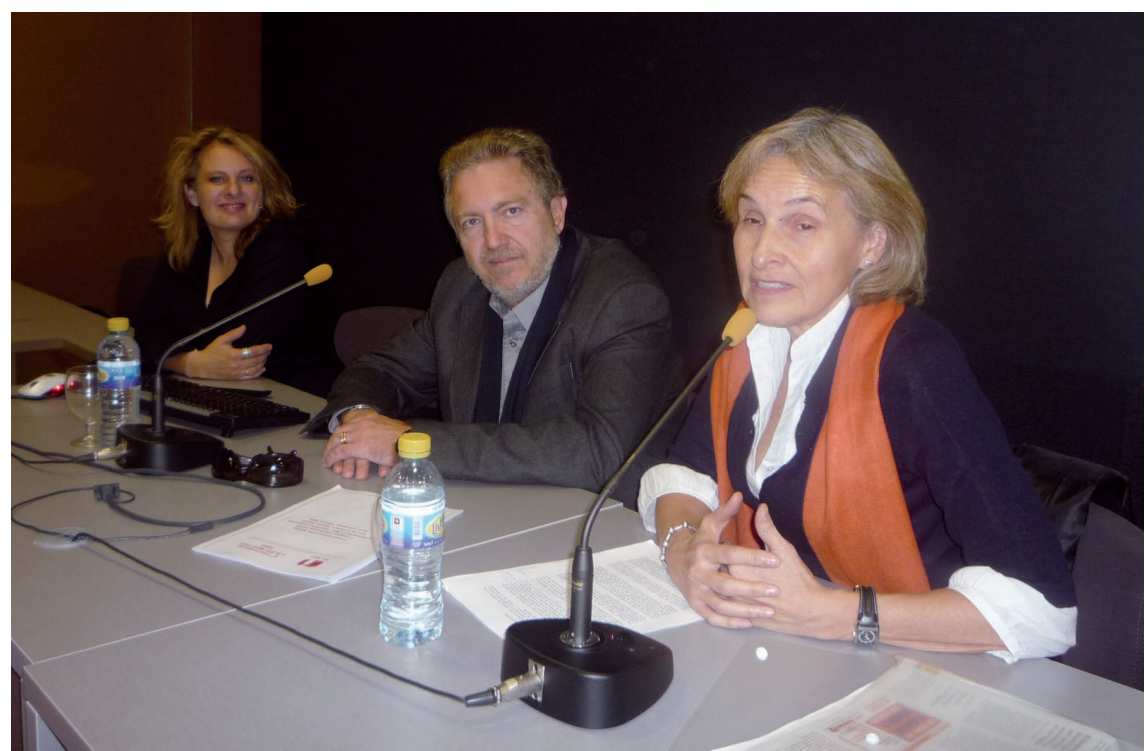

Mesa inaugural: Fernanda Peset, Juan Vicente Botti Navarro y María-Antonia Carrato

El escenario de la información ha cambiado: si bien hace unos años la biblioteca era el agente que servía de intermediario entre ésta y los ciudadanos, ahora este papel ha sido asumido en buena medida por Google. Ante este contexto las bibliotecas tienen dos alternativas: o no hacer nada, asumiendo así y resignándose a la pérdida de importancia de su papel en la sociedad, o examinar la situación actual y reflexionar sobre cómo aprovechar esos cambios para adaptarse al nuevo escenario. En este sentido Morgan plantea algunas posibles actuaciones: crear herramientas que permitan a la comunidad emplear la información, ponerla en contexto y evaluarla de diferentes formas. Para ilustrarlo mostró su catálogo Infomotions $^{1}$. Durante el turno de preguntas los oyentes se interesaron por las habilidades que deberían tener los profesionales de la información, a lo que Morgan respondió sin titubear: "deberían tener conocimientos de xml, indexación, bases de datos relacionales y algo de programación". En su web ${ }^{2}$ puede consultarse el texto de su presentación.

La conferencia de Herget, de la Danube University en Krems (Austria), giró en torno al aprendizaje en la web 2.0 tanto en entornos laborales como de enseñanza. Partió en su disertación de los cambios introducidos por internet en los últimos años, que han supuesto la aparición de un nuevo paradigma, y la actual importancia de la alfabetización informacional como destreza para

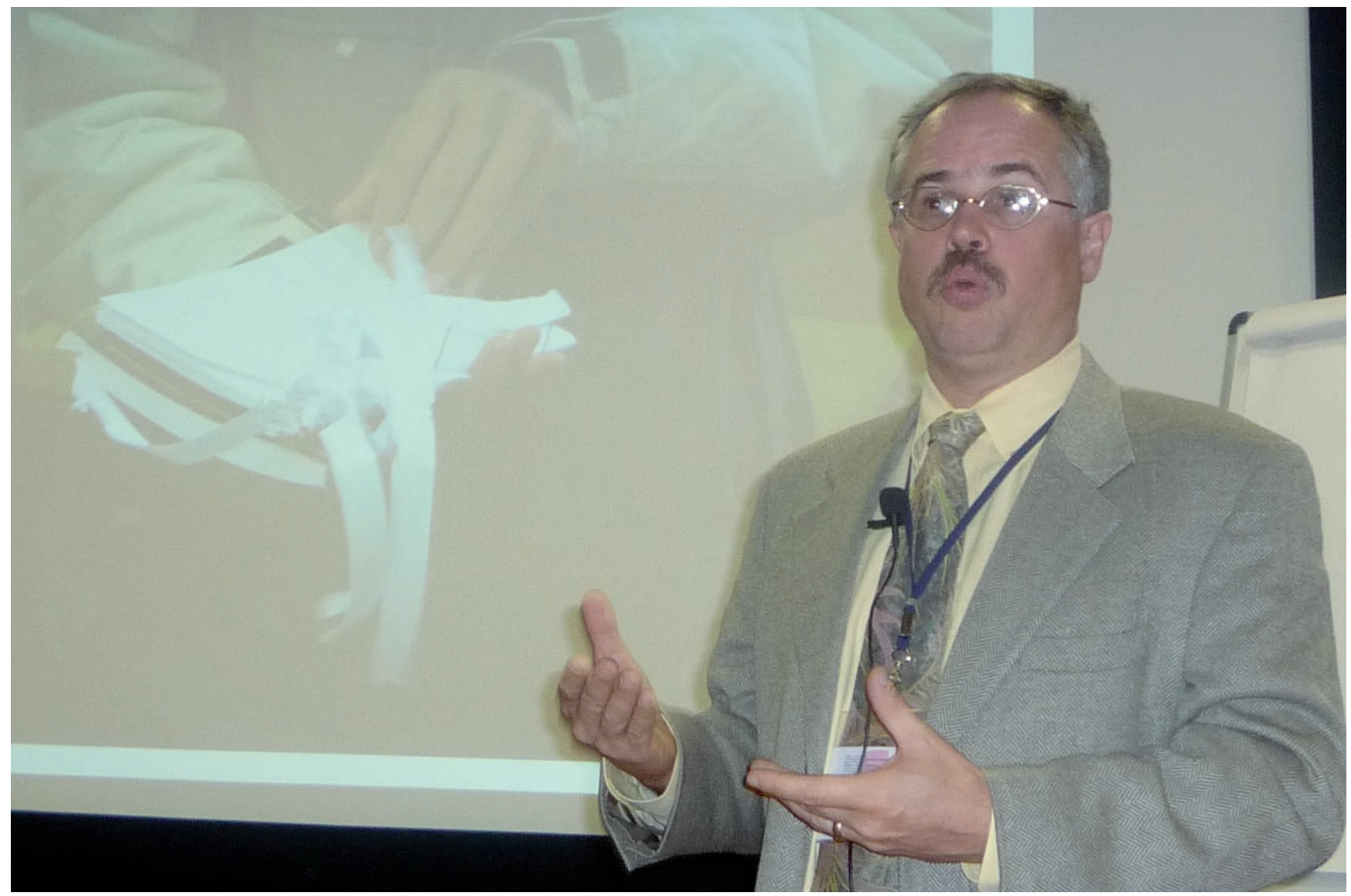

Eric Lease Morgan 


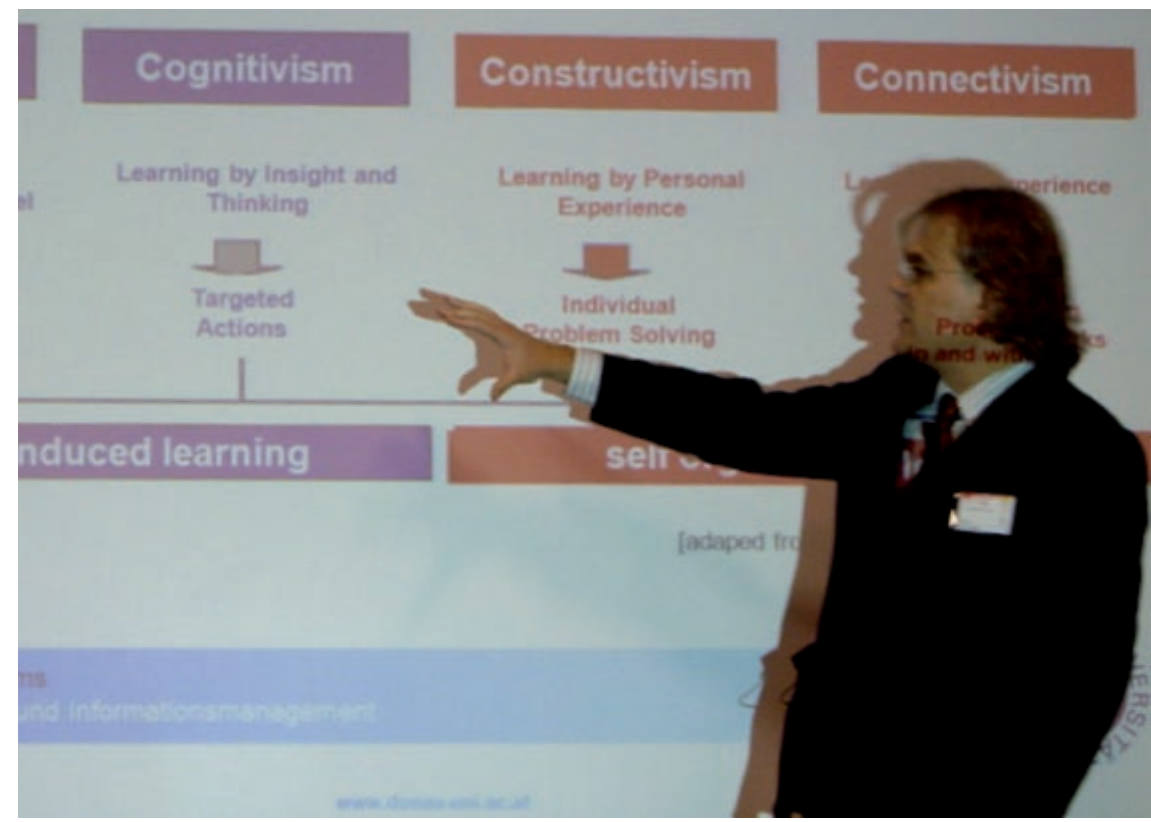

Josef Herget

desenvolverse socialmente. Entroncó con las nuevas teorías conectivistas para valorar muy positivamente el aprendizaje en red basado en la experiencia de otras personas, que nos permite estar constantemente actualizados. En ese contexto es muy interesante el énfasis que puso en la importancia del aprendizaje informal, que supone un $80 \%$, frente al 20\% del formal. Herget propuso también reflexionar acer-

ca de cómo pueden las bibliotecas apoyar este tipo de conocimiento y señaló la necesidad de una orientación en esos procesos de aprendizaje por parte de los usuarios. Quizás el mejor resumen de su interven-

\section{"El conocimiento vive de compartirlo" (Herget)}

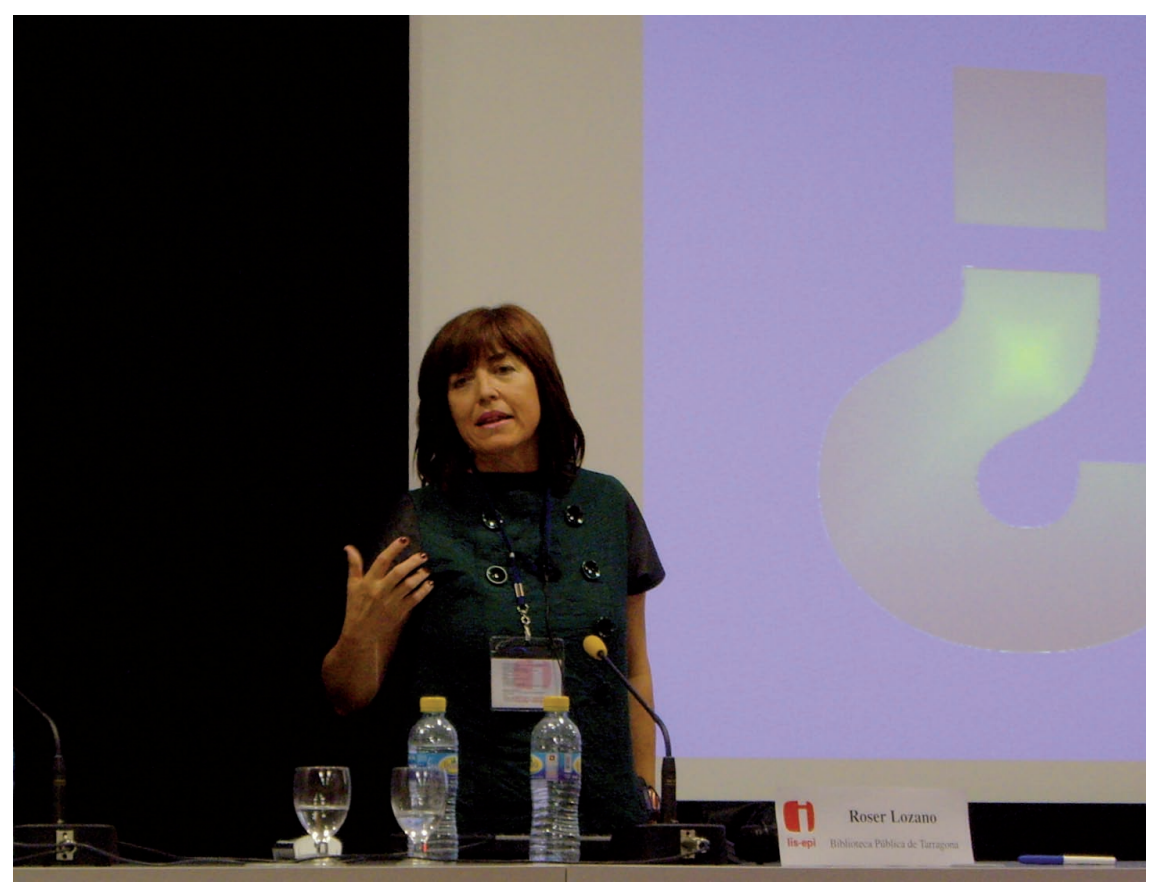

Roser Lozano ción sea una de sus frases finales: "Knowledge lives from sharing" (el conocimiento vive de compartirlo).

En la misma línea temática -el futuro de las bibliotecas ante el cambio tecnológico-, Roser Lozano, todo un referente en el sector bibliotecario español, en la actualidad coordinadora general del CRAI de la Universitat Rovira $i$ Virgili de Tarragona y anteriormente directora de la Biblioteca Pública de Tarragona, habló sobre cómo está afectando dicho cambio al funcionamiento de las bibliotecas. Durante su amena presentación fue desgranando, en el habitual estilo directo con el que escribe sus artículos, multitud de ideas enfocadas especialmente en dos direcciones: las relaciones con los usuarios y el funcionamiento interno. Por ejemplo, consideró que es importante fomentar la participación del personal, ya que sólo así se puede hacer aflorar el talento de las personas e innovar en las organizaciones. Por otra parte, gracias a las herramientas de la web social es posible escuchar a los usuarios, pero también participar en la conversación. Las redes sociales se convierten así en un espacio idóneo para la comunicación con el personal y los usuarios. Coincidiendo con Morgan, Lozano opinó que si las bibliotecas no se adaptan bien al cambio tecnológico quizá no pase nada, pero eso implicará necesariamente una pérdida de su papel en la sociedad. En todo caso los grandes cambios están aún por llegar.

\section{"Fomentar la participación para hacer aflorar el talento de las personas e innovar en las organizaciones" (Lozano)}

Por su parte Luis Collado, representante en España de Google Books, en el plano más genérico del 
consumo de información digital realizó un recorrido por tres aspectos que consideró clave: dispositivos, contenidos y acceso. Enfatizó la importancia de hacer prevalecer los contenidos sobre los dispositivos, dado el carácter efímero e imperfecto de estos últimos. El futuro de los contenidos está, en su opinión, en la digitalización de la información, que permite su indexación y posterior tratamiento y recuperación. Por otra parte la tendencia actual hacia el cloud computing (servicios informáticos en servidores externos) ha sustituido la idea de almacenamiento por la de acceso a la información, como se puede observar en lo que ha sucedido en el mundo de la música.

\section{"La tendencia hacia el cloud computing ha sustituido la idea de almacenamiento por la de acceso a la información" (Collado)}

En su conferencia se echó de menos la referencia a los aspectos más controvertidos o polémicos de Google, como su relación con los editores y demás creadores de contenidos, tanto en el ámbito de la digitalización de libros como en el de los medios de comunicación; sin embargo, aunque él los rehuyera en su exposición inicial, sí aparecieron en el turno de preguntas del público y se comentaron en Twitter.

\section{Rich internet applications, el nuevo paradigma web}

La mesa de debate sobre RIAs, moderada por Isidro F. Aguillo (Cchs-Csic), sirvió para introducir a la audiencia en la novedad que representan este tipo de aplicaciones, sus características, utilidades y perspectivas. David Maniega-

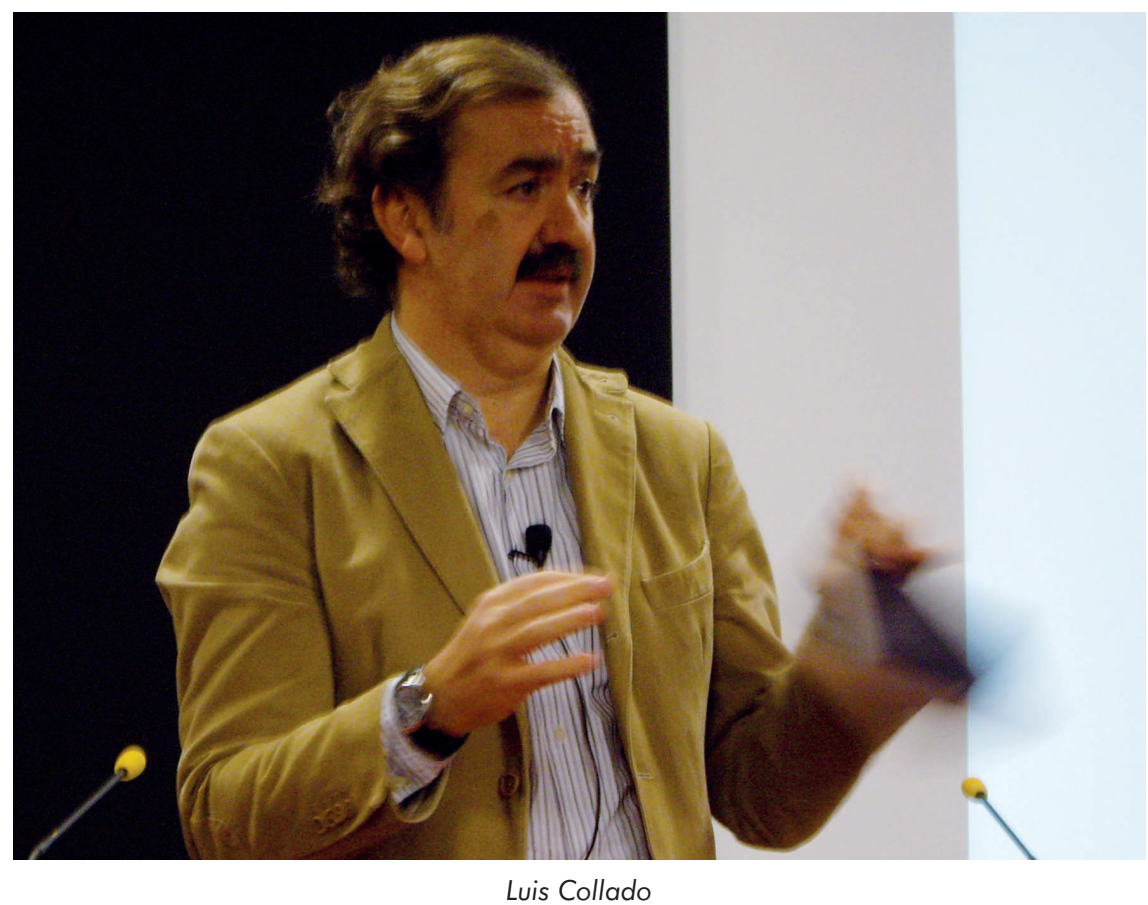

Legarda, de la Universitat Oberta de Catalunya, fue el encargado de poner al público en antecedentes y explicó el modelo RIA y su arquitectura, beneficios, las principales herramientas para la construcción de RIA y seis reglas de oro para su diseño. Entre los retos pendientes indicó: cambios en los hábitos de uso y navegación, asegurar los niveles de accesibilidad, optimización para motores de búsqueda, o su complejidad en sistemas de monitorización, insistiendo especialmente en la importancia de no quedarse en el diseño.

La presentación de Jordi Ceballos (grupo ICA) puso de relieve el nuevo paradigma que suponen las RIAs en la historia de la Web puesto que introducen una mayor riqueza en la interacción con el usuario. Pero su punto de originalidad estuvo en lo que él considera el futuro de las RIAs: las rich mobile applications, en las que se está trabajando en la actualidad y sobre las que aún quedan puntos por resolver. Por último, aunque la intervención de Jordi Castells (Raona) trataba sobre Silverlight y la experiencia de usuario, sin duda será recordada por el rompedor vídeo futurista que mostró ${ }^{3}$. Desde su punto de vista el futuro de las RIAs está en la experiencia de usuario.

\section{"Las RIAs son un nuevo paradigma en la historia de la Web"}

\section{Software libre en bibliotecas: estado de la cuestión y ejemplos prácticos}

La segunda mesa de debate estuvo dedicada al empleo de software libre en bibliotecas, y fue moderada por Ernest Abadal (Universitat de Barcelona), quien recordó cómo hace pocos años estuvo presente en una mesa de la misma temática en la que se habló de algunos proyectos pero de pocas realidades, mientras que en estos momentos ya se pueden presentar ejemplos reales. Patricia Russo $(\mathrm{Cobdc})$ realizó una panorámica del estado del arte en la actualidad, repasando los tipos de software libre para bibliotecas, con sus funciones más características y ejemplos de programas, indicando 


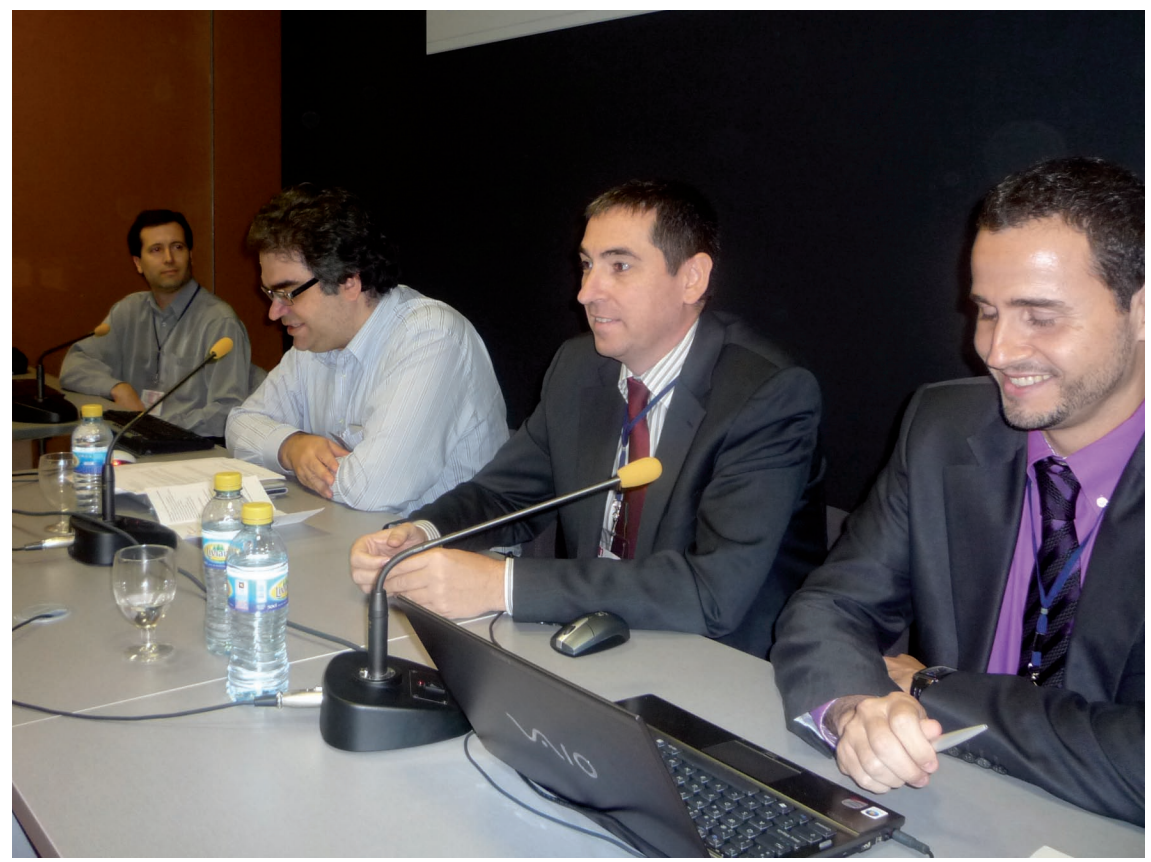

Mesa de RIAs: Jordi Ceballos, Isidro F. Aguillo, Jordi Castells y David Maniega

algunas perspectivas de futuro y proponiendo un ejemplo factible de biblioteca funcionando completamente con software libre.

Los casos prácticos estuvieron a cargo de los dos Serrano de la mesa: Jordi Serrano (Universitat Politécnica de Catalunya) y Jorge Serrano-Cobos (MASmedios). El primero explicó cómo se ha abordado recientemente la actualiza- ción del portal Bibliotécnica de las bibliotecas de la $U P C^{4}$ con el cms Drupal, escogido tras una fase de testeo en la que se tuvieron en cuenta varios objetivos como la creación de un portal 2.0 que pueda englobar todas las herramientas de la universidad (opac, depósito, servicios...) y que pueda facilitar la recuperación de la información. La disyuntiva más importante se planteó en la

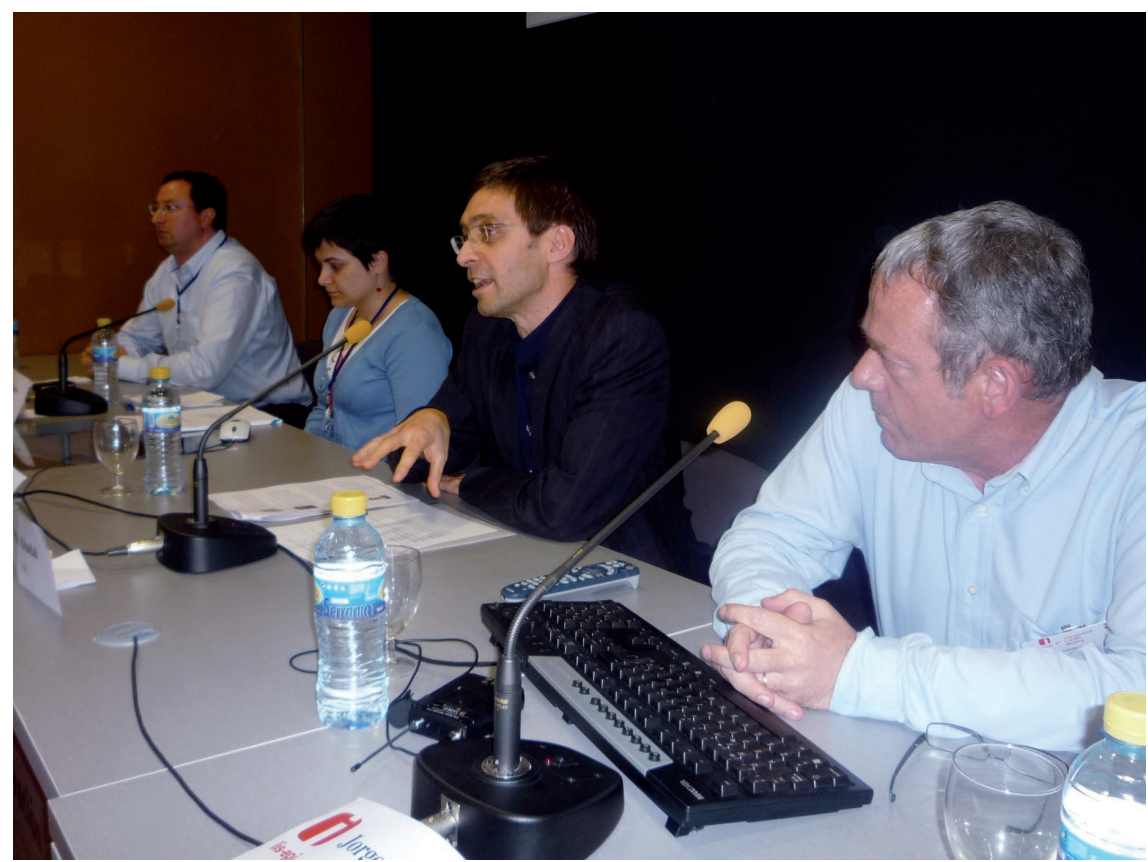

Mesa de software libre y bibliotecas: Jorge Serrano-Cobos, Patricia Russo, Ernest Abadal y Jordi Serrano integración de esta herramienta con el catálogo actual que funciona con el software comercial Millennium y el servidor de OAI, en DSpace. Serrano destacó asimismo los resultados finales, tanto positivos como negativos, y las perspectivas para mejoras de futuro.

Serrano-Cobos presentó el catálogo Infobook que su empresa ha desarrollado para el $B B V A$ con el sistema de gestión bibliotecaria Koha, manteniendo a la audiencia en vilo con la rapidez y precisión de su exposición. Desde su punto de vista una de las grandes bazas del software libre en el desarrollo de proyectos son sus amplias funciones de personalización, y manifestó el interés de Koha como sistema de gestión bibliotecaria de código abierto por las posibilidades de innovación que ofrece. La significación de su ponencia reside en que Infobook constituye uno de los pocos ejemplos en nuestro país de auténtico opac social.

\section{"Infobook constituye uno de los pocos ejemplos en nuestro país de auténtico opac social" (Serrano-Cobos)}

\section{Acceso abierto y evaluación de la investigación}

El ámbito científico estuvo representado por las iniciativas de acceso abierto expuestas en una de las mesas de debate, y por la conferencia de Félix de Moya que puso punto y final al encuentro.

La mesa de debate contó con una amplia representación del equipo de $E$-Lis, el repositorio internacional sobre biblioteconomía y documentación. Por una parte, Julio Alonso-Arévalo, de la Uni- 
versidad de Salamanca, repasó los seis años de funcionamiento de este repositorio reflejados en un análisis de sus estadísticas de uso en el que España se reveló como el país con más depósitos y el tercero con más consultas.

Un aspecto crucial en un repositorio internacional como E-Lis, como es el de su financiación, fue el tema de la intervención de Fernanda Peset (Universidad Politécnica de Valencia), que contó cómo se han ido resolviendo los problemas de financiación de E-Lis y ofreció algunos consejos para proyectos de características similares.

\section{"España es el país con más depósitos y el tercero con más consultas en E-Lis"}

Otras intervenciones fueron las de Nuria Solé-Bardalet y Linda Valdés, que presentaron el trabajo de la Fundació Tàpies en Open Access en una herramienta para la difusión de contenidos culturales desarrollada desde esta fundación, y de Paula Sequeiros, quien abordó las relaciones de poder en el ámbito de la comunicación científica y el papel de los repositorios en ese escenario.

Félix de Moya expuso el trabajo del grupo de investigación SCI$m a g o^{5}$ que él dirige, y algunos de sus proyectos en curso: el SCImago Institutions Rankings y el SCImago Journal \& Country Rank. El primero es un ranking de más de 2.000 instituciones dedicadas a la investigación, a través del cual Moya nos mostró algunas de las características del sistema universitario español, como la homogeneidad de la distribución de las universidades y el hecho de que realmente ninguna de ellas alcanza un cierto grado de

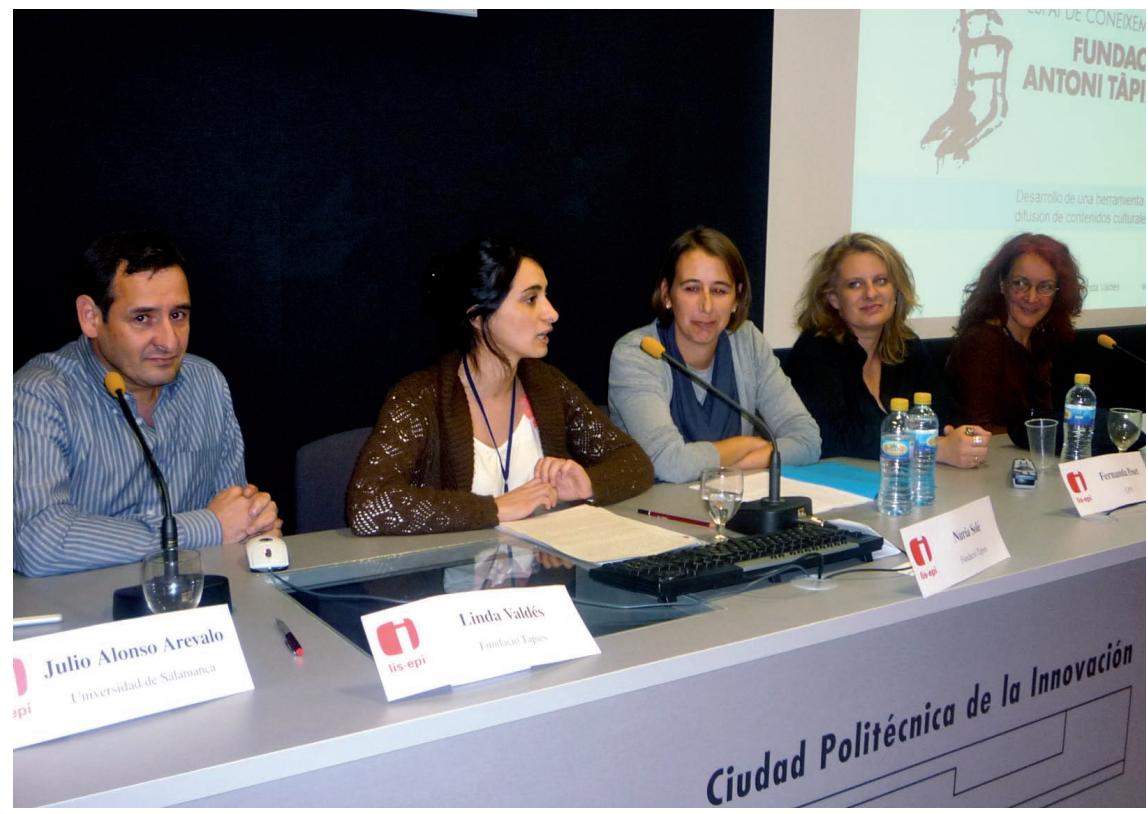

Mesa de acceso abierto: Julio Alonso-Arévalo, Linda Valdés, Nuria Solé-Bardalet, Fernanda Peset y Paula Sequeiros

excelencia, al menos según los parámetros utilizados.

SCImago Journal \& Country Rank $^{6}$ es una herramienta abierta para el análisis de la ciencia que muestra los indicadores científicos de revistas y países a partir de los datos contenidos en la base de datos Scopus, de Elsevier. Uno de esos indicadores es el SCImago Journal Rank (SJR), que mide la visibilidad de las revistas en Scopus y utiliza el mismo algoritmo PageRank de Google. En comparación con el Factor de impacto de ISI Thomson, para cuyo cálculo valen igual todas las citas, el $S J R$ realza la posición de las mejores revistas, puesto que valen más las citas provenientes de las revistas más citadas.

\section{"En el cálculo del factor SJR se valoran más las citas provenientes de las revistas más citadas"}

Para ilustrar su funcionamiento, mostró el módulo de publicaciones científicas, con el ejemplo de El pro- fesional de la información, y cómo emplearlo para comparar los resultados de investigación de diferentes países y generar mapas visuales. En el turno de preguntas los asistentes se interesaron por el papel de los repositorios y del acceso abierto en la evaluación científica, y el uso del $S J R$ en el proceso de evaluación de la Aneca.

\section{Dispositivos móviles para todo}

El bloque dedicado a los aparatos móviles actuales y futuros de acceso a la información se inició con la intervención del especialista en periodismo digital Javier DíazNoci (Universitat Pompeu Fabra), quien trazó el origen, evolución y tendencias de futuro de los diferentes dispositivos móviles existentes para la lectura de medios de comunicación. Recordó algunos fallidos prototipos realizados a mediados de los años noventa del siglo pasado, como los de Roger Fidler, o en nuestro país el proyecto Newspad de El periódico de Catalunya, para desgranar a continuación de manera pormenorizada las diferentes experiencias actuales y tendencias futuras de dispositivos móviles para prensa, desde teléfonos móviles a $e$ - 


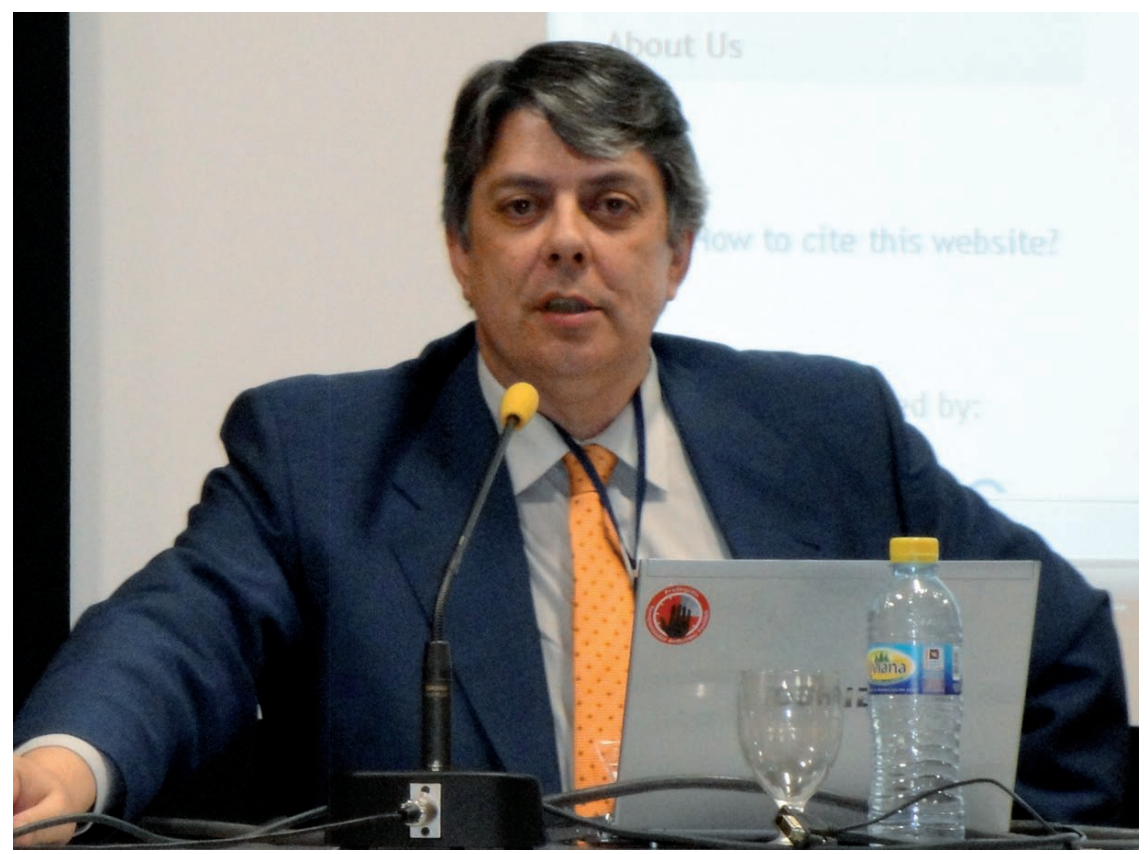

Félix de Moya

readers como los modelos de Sony y el Kindle de Amazon. El debate -inevitable en el momento actualsobre la crisis de los medios de comunicación y el futuro de la prensa se alargó con las intervenciones de los presentes.

A continuación, la feria de dispositivos móviles (Living Lab) que organizó Pablo Lara-Navarra $(U O C)$, buscó la participación de los asistentes para que probaran algunos de los aparatos que trajo para la ocasión, para lo que fue solicitando varios turnos de voluntarios mientras tenían lugar las charlas de José-Ángel Martínez-Usero (Technosite) y la suya. Martínez-Usero dedicó su tiempo a explicar el proyecto Inredis ${ }^{7}$, subrayando la importancia de crear tecnologías accesibles e interoperables, orientadas a las personas. El punto práctico lo marcó una demostración en la que se emulaba el uso de un cajero automático accesible para personas con discapacidad visual.

Lara-Navarra mostró una vistosa presentación en vídeo en la que apostó, al igual que su compañero, por la accesibilidad y la interoperabilidad. Siguiendo el método prác- llamativas, como las dificultades de algunos voluntarios para emplear algunos de los aparatos sin haber recibido instrucciones previas: por ejemplo, algunos habían considerado que el aparato de tinta electrónica estaba encendido cuando estaba apagado.

\section{"La open innovation propone la innovación teniendo en cuenta las opiniones de los usuarios"}

\section{A modo de balance}

Algunas de las conclusiones tico mostró la realidad aumentada, un sistema que permite superponer datos virtuales a la visión del mundo real, creando así una realidad mixta. En consonancia con la charla de Roser Lozano introdujo el concepto de open innovation, que propone la innovación teniendo en cuenta las opiniones de los usuarios. Para terminar, Lara interpretó los resultados de las pruebas de uso de dispositivos móviles realizadas por los voluntarios. Hubo conclusiones que se pudieron extraer de las ponencias y debates dibujan el futuro inmediato de la información en base a algunos elementos clave: la web como principal plataforma de acceso a la información; el uso de nuevos dispositivos para consultarla, con una característica en común: la movilidad; aplicaciones web mucho más ricas; consolidación del acceso abierto, así como del software libre como alternativa cada vez más sólida ante el software propietario.

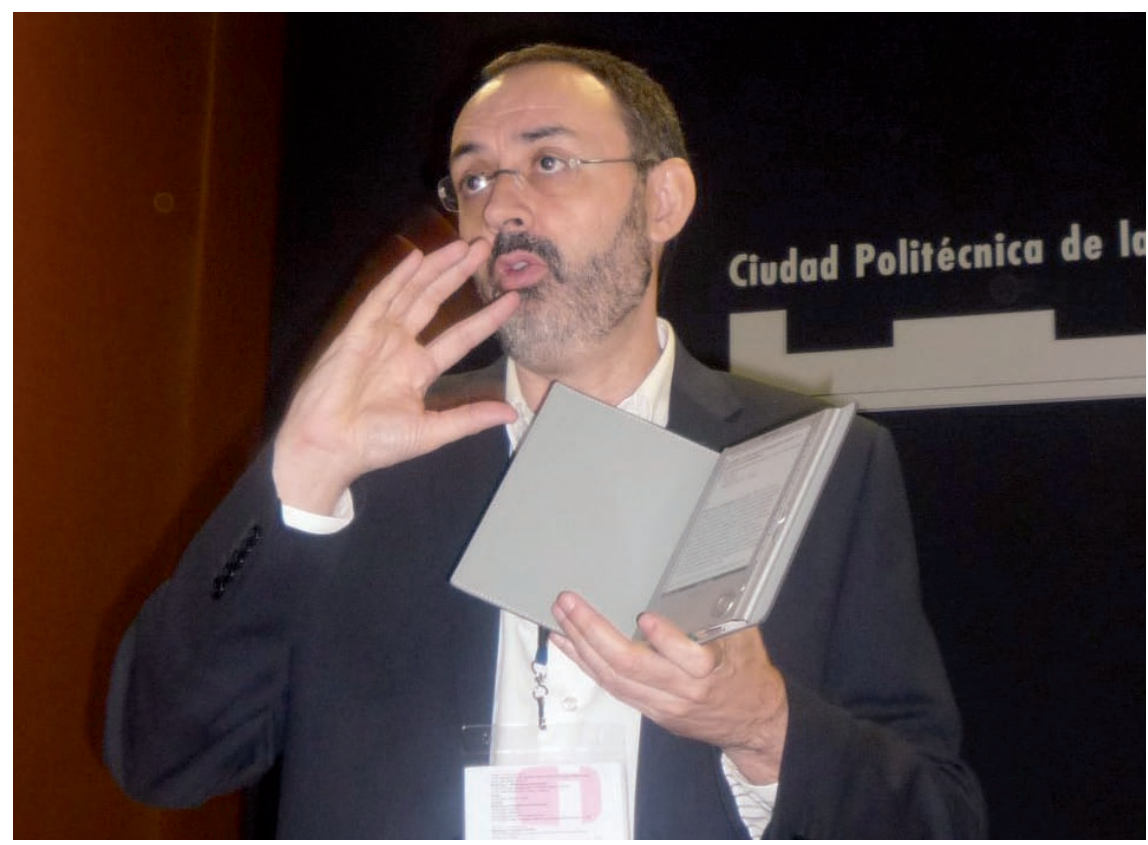

Javier Díaz-Noci comentando un e-reader 
Todo ello conlleva la adaptación de las organizaciones -como las bibliotecas- a los cambios tecnológicos, lo que requiere a su vez un cambio de mentalidad.

En definitiva, un encuentro en el que se mostraron algunas de las principales preocupaciones actuales de la profesión y de las líneas en las que se trabajará con toda seguridad en los próximos años.

Más información en los enlaces oficiales del evento:

Web

http://www.ciepi.org/lisepi09/index. html

\section{$\mathrm{B} \log$}

http://lisepi09.wordpress.com/

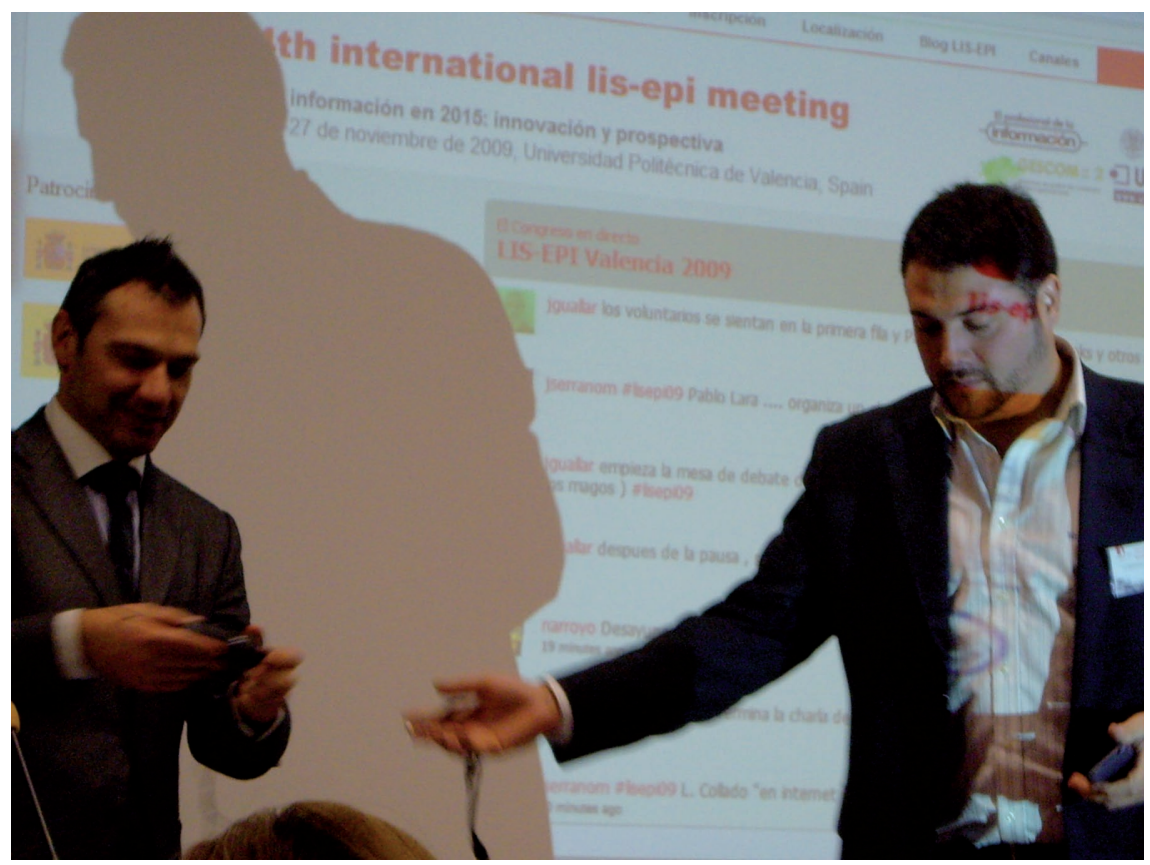

José-Ángel Martínez-Usero y Pablo Lara-Navarra

\section{Notas}

1. http://infomotions.com/alex

2. http://infomotions.com/musings/future-2015/

3. http://www.youtube.com/watch? $v=p z S x b L l M$ $o G Y$
4. http://bibliotecnica.upc.edu/
5. http://www.scimago.es/
6. http://www.scimagojr.com/
7. http://www.inredis.es/

\section{Referencias}

Redondo, Sílvia. "Redes, publicación científica e innovación en el LIS-EPI Meeting 2008". El profesional de la información, 2008, noviembrediciembre, v. 17, n. 6, pp. 685-688.

\section{Natalia Arroyo-Vázquez natalia.arroyo@gmail.com}

Javier Guallar

jguallar@gmail.com

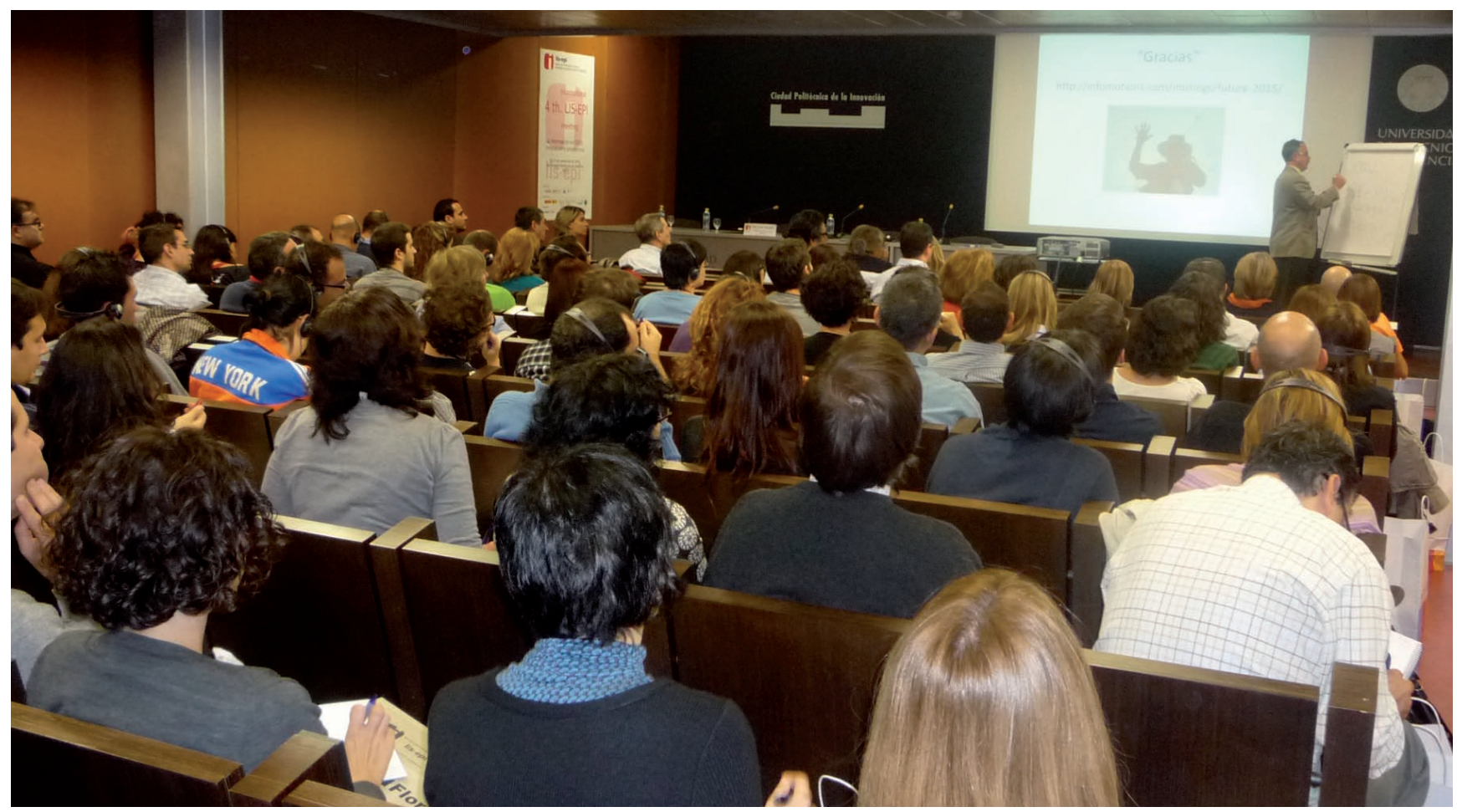

Asistentes al meeting 